American Journal of Infectious Diseases 3 (2): 110-114, 2007

ISSN 1553-6203

(C) 2007 Science Publications

\title{
Emetine produce Entamoeba histolytica Death by Inducing a Programmed Cell Death
}

\author{
${ }^{1}$ J.D. Villalba-Magdaleno, ${ }^{1}$ R. Rojas, ${ }^{1}$ C. Gómez, ${ }^{2}$ M. Shibayama, ${ }^{3}$ Julio C. Carrero and ${ }^{1}$ D.G. \\ From the ${ }^{1}$ Programa de Biomedicina Molecular ENMyH; Instituto Politécnico Nacional, Guillermo \\ Massieu Helguera 239, CP 07320, México, DF, México; ${ }^{2}$ Departamento de Patología Experimental \\ CINVESTAV-IPN Ap postal 14-740, CP 07300, México DF, México; ${ }^{3}$ Departamento de Inmunología, \\ Instituto de Investigaciones Biomédicas, UNAM, México, DF, México
}

\begin{abstract}
Amoebiasis is a major public health problem in tropical and subtropical countries. Although a number of antiamoebic agents are used for its treatment and the molecular targets of these drugs have been identified, the biochemical and physiological mechanisms that produce trophozoite death are little known. The present study dates the in vitro induction of programmed cell death (PCD) in E. histolytica by emetine. Morphologically, the emetine incubation reduces cytoplasmic volume, produce nuclear condensation and DNA fragmentation, maintaining the plasma membrane integrity; biochemically, the morphological changes are in concordance with the overproduction of reactive oxygen species inside trophozoites. These results provide the first insight related the cellular mechanisms of emetine action.
\end{abstract}

Key words: Entamoeba histolytica, Programmed Cell Death, Emetine, Apoptosis

\section{INTRODUCTION}

Entamoeba histolytica, the causal agent of amoebiasis, is a parasite with a remarkable ability to kill host cells. Several efforts have been concentrated on understanding this phenomena and how it participates in pathogenesis. Amoeba kills different host cell types, including neutrophils, erythrocytes, lymphocytes, and epithelial cells ${ }^{[1,2,3]}$. Boettner and Petri have proposed that pathogenesis occurs in three steps: i) the amoeba contact and adheres the host cells trough specific lectins ${ }^{[5]}$, ii) the amoeba produces morphological and biochemical changes in the host cells, including cell shrinkage, intracellular calcium efflux, caspase activation and phosphatidylserine exposition $^{[4]}$, and iii) the parasite engulf target cells ${ }^{[6]}$. The evidences suggest that the induction of an apoptotic process could be one of the mechanisms used by the amoeba to kills the host cells.

In metazoan, apoptosis, a form of programmed cell death (PCD), have evolved not only to regulate growth and development, but also to protect the cell against viral, bacterial, or parasitic infections ${ }^{[7,8]}$. In addition, this form of cell death leads to the elimination of undesired cells without inducing any inflammatory response ${ }^{[9]}$. PCD include biochemical changes such as caspase activation, exposition of phosphatidylserine in the plasma membrane, intracellular $\mathrm{Ca}^{+2}$ increment, mitochondrial dysfunction, as well as physical changes such as cell shrinkage, chromatin condensation and DNA fragmentation ${ }^{[18,11]}$.
Increasing evidences indicates that PCD can also be present in unicellular eukaryotes, such as yeast and several protozoans including the parasites Trypanosoma, Leishmania and Plasmodium [12-14]. Recently, our group reported the morphological and biochemical characteristics of PCD in E. histolytica induced by G418 (paper in editorial review).

Amoebiasis is primarily controlled by pharmacological treatment of symptomatic individuals using metronidazole, emetine and halogenated hydroxyquinoline. Although the molecular targets that interact with these drugs have been described, little is know about the biochemical and physiological effects that provokes amoeba death. Emetine is an alkaloid that inhibits eukaryotic protein synthesis by blocking translocation of the peptidyl-tRNA from acceptor to donor site. In human leukemia cells has been reported that emetine and cycloheximide induce apoptosis, correlating with the appearance of large (20 to $\square 580$ kilobase pairs) DNA fragments ${ }^{[15]}$. Here to study the cellular effects of emetine in the death of E. histolytica trophozoites, we characterized the morphological and biochemical changes induced by the drug. The results suggest that a Programmed Cell Death mechanism is induced by emetine.

\section{METHODS}

Parasite Culture and Maintenance. Trophozoites of clone A (strain HM1:IMSS) were axenically cultured in TYI-S-33 medium ${ }^{[16]}$. Trophozoites induced to PCD

Corresponding Author: Dr. D. Guillermo Pérez Ishiwara (Ph. D),Program of Molecular Biomedicine, ENMyH-IPN. Guillermo Massieu Helguera \#239. Fracc "La Escalera", Ticomán, CP 07320, México, D.F. Tel/fax (55) 55864741 
were incubated with $120 \mu \mathrm{g} / \mathrm{ml}$ emetine at different periods of time as indicated bellow.

Kinetics of Growth. Growth curves of trophozoites were done in the absence or in the presence of 120 and $240 \mu \mathrm{g} / \mathrm{ml}$ emetine. Trophozoites viability was measured each $12 \mathrm{~h}$ by trypan blue exclusion.

Microscopy Analysis. Trophozoites were washed twice with PBS and placed on glass slides. Trophozoites were fixed in $2 \%$ formaldehyde and observed in an Olympus BX41 inverted microscope coupled to a video digital capture CoolSNAP-Pro Media Cybernetics, using Image-pro Plus software.

In Situ Cell Death Detection (TUNEL). Ten thousand emetine-treated or untreated trophozoites were fixed in $4 \%$ formaldehyde for $45 \mathrm{~min}$ at $4{ }^{\circ} \mathrm{C}$. After washing two times with PBS, $50 \mu$ terminal deoxynucleotidyl transferase-mediated biotin-dUTP nick end labeling (TUNEL) reaction mixture (ROCHE) was added and incubated for $60 \mathrm{~min}$ at $37{ }^{\circ} \mathrm{C}$ in a humidified atmosphere in the dark. Trophozoites were rinsed three times with PBS, loaded on slides, and observed with a Zeiss ESM-Pascal confocal microscope. As a positive control, trophozoites were treated with $20 \mu \mathrm{g} / \mu \mathrm{l}$ DNAse I endonuclease for $10 \mathrm{~min}$.

Transmission Electron Microscopy. Trophozoites grown in the presence and absence of 543emetine were washed twice with $0.1 \mathrm{M}$ sodium cacodylate buffer and fixed for $1 \mathrm{~h}$ with $2.5 \%$ glutaraldehyde in $0.1 \mathrm{M}$ sodium cacodylate buffer ( $\mathrm{pH}$ 7.4). Fixed trophozoites were washed twice with $0.1 \mathrm{M}$ sodium cacodylate buffer, postfixed with $2.0 \%$ osmium tetroxide, dehydrated with ethanol at increasing concentrations, and finally with propilen oxide. The trophozoites were then embedded in Epoxy resins. Semithin sections were stained with toluidine blue for light microscope examination. Thin sections were stained with uranyl acetate followed by lead citrate and examined using a Zeiss EM-10 electron microscope.

Measurement of Radical Oxigen Species Level (ROS). To determine the level of ROS, the cellpermeant probe dichlorodihydrofluorescein (DCFDA) was used. In the presence of a proper oxidant, it is oxidized to the highly fluorescent 2,7dichlorofluorescein. $1 \times 10^{6}$ emetine treated or untreated trophozoites were resuspended in $500 \mu \mathrm{l}$ of phosphate buffer $\mathrm{pH} 7.4$, containing $20 \mu \mathrm{M}$ DCFDA, incubated in dark for $15 \mathrm{~min}$ and analyzed by flow cytometry using CELLQuest software (Becton Dickinson, San Jose, CA) at 485 and $525 \mathrm{~nm}$ excitation and emission, respectively.

\section{RESULTS}

Citotoxic effect of emetine on E. histolytica trophozoites. The effect of emetine on trophozoites viability was assessed by trypan blue exclusion staining. The results showed that $87 \%$ of trophozoites incubated with 120 and $240 \square \mathrm{g} / \mathrm{ml}$ emetine died after $48 \mathrm{~h}$ (Fig 1A). By light microscopy analysis we observed that non-treated parasites (NT) had typical amoebic forms, while trophozoites exposed to emetine showed rounded forms and cell shrinkage (Fig 1B). To determine whether emetine induces trophozoite shrinkage, trophozoite size was measured by flow cytometry analysis. As shown in Fig. 1C, the trophozoite population treated with emetine showed a marked reduction in size, in comparison with NT trophozoites. While $47.2 \%$ of NT trophozoites remain over the mean value size, the $85.2 \%$ of emetine treated parasites diminished in size. Interestingly, changes in granularity were also observed by the increase in side scatter, from $16.8 \%$ in NT trophozoites to $52.5 \%$ in emetine-treated parasites. These morphological changes resemble those observed during PCD. Thus, we proceed to study the biochemical changes induced by emetine, analyzing the nuclear and cytosolic effects associated with a putative PCD process.
A

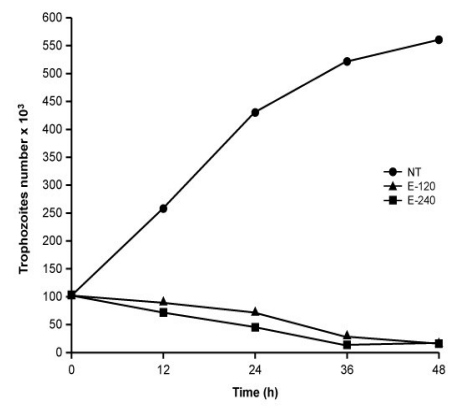

C
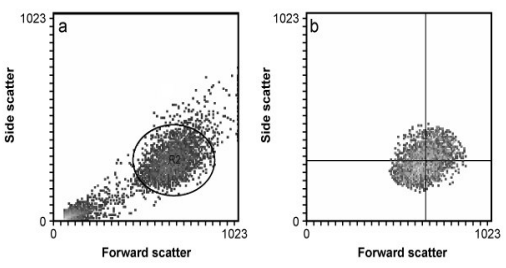
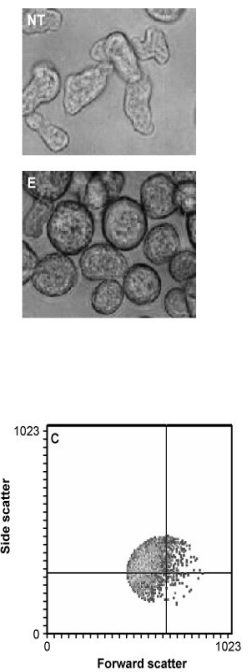

Fig. 1: Effects of emetine in the viability and morphology of E. histolytica. A) Grow kinetics of trophozoites cultured in the absence (NT) or in the presence of 120 and $240 \mu \mathrm{g} / \mathrm{ml}$ of emetine (E-120 or E-240). Viability was evaluated by trypan blue exclusion. $B$ ) Contrast phase micrographs showing the morphology of non- treated (NT) and emetine treated (E) trophozoites. $C$ ) Representative dot plots of flow cytometry showing cell size (forward scatter) and granularity (side scatter) of NT (b) and emetine treated (c) trophozoites. The trophozoites population analyzed derived from the indicated cytogram gate R2 (a). 
Emetine induces DNA fragmentation in E. histolytica. In mammalian cells, internucleosomal DNA fragmentation is one of the most important and typical nuclear features that define the PCD phenomenon ${ }^{[17]}$. To confirm DNA fragmentation, TUNEL assays were conducted. Less than $5 \%$ of nuclei of NT trophozoites were stained (Fig. 2A). In contrast, seventy percent of trophozoites showed positive nuclear staining after $24 \mathrm{~h}$ of emetine incubation. In fact staining spots within the nuclei evidenced the DNA fragmentation. By transmission electron microscopy we observed several changes in the E. histolytica nuclei. The nucleus of NT trophozoites showed a dense peripheral chromatin with a central "endosome" and the nuclear and plasma membranes appeared intact. However after $9 \mathrm{~h}$ incubation of trophozoites with emetine, the chromatin was fragmented and displaced to one side of the amoeba nucleus. Cytoplasm showed large vacuoles and the amount of glycogen increased. After $12 \mathrm{~h}$ of emetine interaction, a smaller nucleus was occupied by fragmented and dense chromatin and the round nuclear bodies were more conspicuous. The out limit of the nuclear envelope was not clearly defined. Cytoplasmic glycogen increased significantly and the number and size of vacuoles substantially increased. In all the times studied, the integrity of cytoplasmic membranes remains normal (fig. 2B).
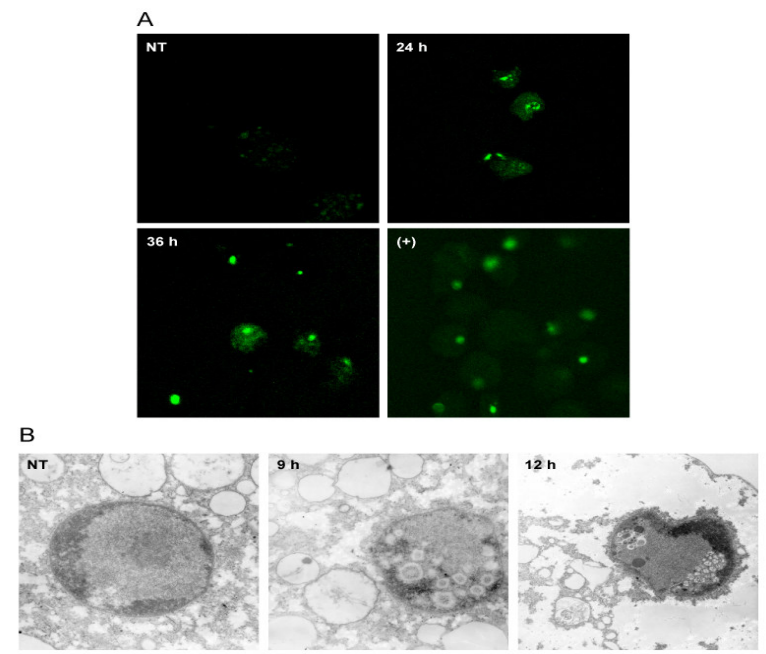

Fig. 2: DNA fragmentation and ultrastructural changes in trophozoites after emetine treatment. A) Confocal microscopy analysis showing nuclear TUNEL staining of NT trophozoites and after 24 or $30 \mathrm{~h}$ of emetine incubation. As a positive control, trophozoites were treated with $20 \mu \mathrm{g} / \mu \mathrm{l}$ DNAse I (+). B) Transmission electron micrographs showing the ultrastructure of NT or emetine treated trophozoites after $9 \mathrm{~h}$ or $12 \mathrm{~h}$ of incubation.
Emetine induces oxidative stress in E. histolytica trophozoites. To determine if the generation of internal reactive oxygen species (ROS) is associated to PCD, we analysed the production of ROS in trophozoites treated with $120 \mu \mathrm{g} / \mathrm{ml}$ emetine, determining by flow cytometry the conversion of the DCFDA compound to the highly fluorescent 2, 7 -dichlorofluorescein in the presence of a proper oxidant. As it can see in fig 3, NT parasites displayed basal ROS signals (Fig. 3 continuous line), whereas the emetine treated parasites produced an important enhancement of ROS production, $96 \%$ of trophozoites population showed 10 folds increment of the basal ROS fluorescence (Fig. 3 dashed line)

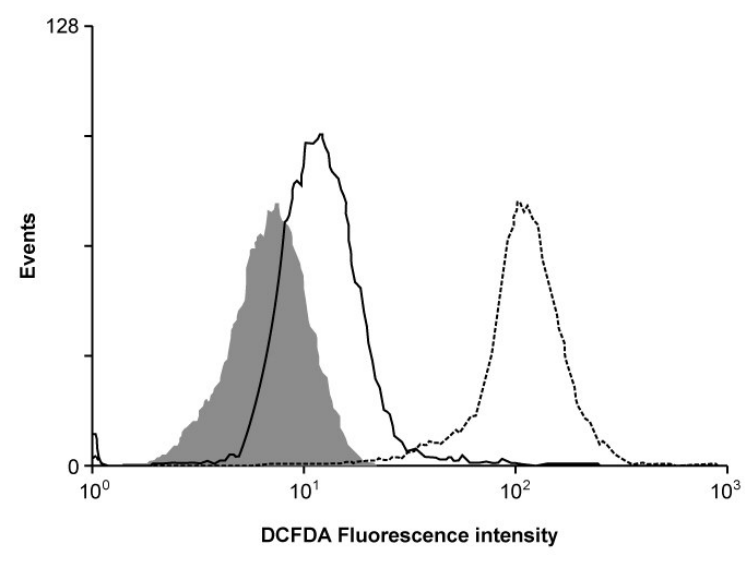

Fig. 3: Detection of reactive oxygen species (ROS). ROS in NT (continuous line) or emetinetreated (dashed line) trophozoites were measured by flow cytometry using the fluorescent dye DCFDA. The shaded histogram peak represents the negative control fluorescence.

\section{DISCUSSION}

As with other parasitic infections, amoebiasis is a consequence of poverty and bad sanitary conditions. Unfortunately, with the exception of malaria, no vaccines against of protozoan parasites are insight, and poor countries cannot afford to finance large preventive medicine programs. Amoebiasis is controlled primarily by drug treatment of symptomatic individuals using metronidazole, emetine, chloroquine and iodoquinol. Till today, emetine remains as one of the best drugs for treating amoebic liver abscess ${ }^{[18,19]}$. Emetine, a plant alkaloid, kills the trophozoites of $E$. histolytica mainly by inhibiting protein synthesis. At 
molecular level there is an irreversible, but noncovalent binding to the peptide-chain elongation site of the $60 \mathrm{~S}$ subunit of ribosomes ${ }^{[20]}$. The inhibition of eukaryotic protein synthesis activity by emetine has been linked to its capacity to induce apoptosis in numerous mammal cell types ${ }^{[15,21,22]}$. However, it is not yet established whether emetine can induce PCD in E. histolytica parasite. There are now increasing number of reports regarding unicellular organisms undergoing apoptosislike death, whose induction can be activated under threatening circumstances. In an earlier study, we described similar apoptotic-like features in this organism in response to the antibiotic G418 (paper in editorial review). Here we showed that treatment with $120 \square \mathrm{g} / \mathrm{ml}$ emetine produced cell shrinkage. Flow cytometry data further supported cell shrinkage observed under light microscopy, representing an important morphological evidence of PCD. On the other hand, TUNEL assays indicated that emetinetreated trophozoites undergo in situ DNA fragmentation up $24 \mathrm{~h}$. TUNEL detects $3^{\prime}-\mathrm{OH}$ groups at the end of single-strand and double-strand DNA cuts, suggesting that even though no agarose ladder bands were evident (data not shown), the internal nicked DNA in nuclei trophozoites, evidenced one of the most important characteristics of PCD phenomenon. Transmission electron micrographs of emetine-treated trophozoites clearly showed ultrastructural alterations related to PCD. Similar alterations have been shown in Dictyostelium discoideum ${ }^{[23]}$ and Blastocystis hominis [24], exhibiting the features of PCD such as cell shrinkage, nuclear condensation, heavy vacuolization, not ladder-like fragmentation, and the maintenance of plasma membrane integrity.

Biochemically, emetine induced the production of ROS. The overproduction of ROS species could cause an increase in the level of lipid peroxidation damage ${ }^{[25]}$. In general, the overall effect of lipid peroxidation is to decreased membrane fluidity, as well as increase leakiness of the membrane, leading to complete loss of membrane integrity ${ }^{[26]}$. This in turn causes an increase in intracellular $\mathrm{Ca}^{2+}$ level inside cells, lack of $\mathrm{K}^{+}$and the release of protons into the cytosol contributing to the intracellular acidification.

In conclusion, our observations suggest that the inhibition of protein synthesis produced by emetine exert a PCD mechanism in E. histolytica trophozoites. The results present here, together with our previous publications lead us to speculate that PCD mechanisms in E. histolytica have several morphological and biochemical similarities to higher eukaryotes, and that it could be induced by several chemical stresses. Currently, we are employing molecular methods to identify genes that induce the PCD mechanism as well as genes that protect trophozoites against PCD.

\section{ACKNOWLEDGEMENTS}

The authors gratefully acknowledge Angelica SilvaOlivares for excellent technical assistance in transmission electron microscopy, and we also thank Alfredo Padilla Barberi for the graphical art design.

\section{REFERENCES}

1. Guerrant, R. L., J. Brush, J.I. Ravdin, J.A. Sullivan, and G.L. Mandell, 1981. Interaction between Entamoeba histolytica and human polymorphonuclear neutrophils. J. Infect. Dis. 143: 83-93.

2. Salata, R. A., J. G. Cox, and J. I. Ravdi, 1987. The interaction of human T-lymphocytes and Entamoeba histolytica: killing of virulent amoeba by lectin-dependent lymphocytes. Parasite Immunol. 9: 249-261.

3. Kobiler, D., and D. Mirelman, 1981. Adhesion of Entamoeba histolytica trophozoites to monolayers of human cells. J. Infect. Dis. 144: 539-546.

4. Boettner, D.R., and W.A. Petri, 2004. Entamoeba histolytica activates host cell caspases during contact-dependent cell killing. Curr. Top Microbiol. Immunol. 289: 175-184.

5. Mann, B.J. 2002. Structure and function of the Entamoeba histolytica Gal/GalNac lectin. Int. Rev. Cytol. 216: 59-80.

6. Huston, C.D., D.R. Boettner, V. Miller-Sims and W.A. Petri, Jr., 2003. Apoptotic killing and phagocytosis of host cells by the parasite Entamoeba histolytica. Infect. Immun. 71: 964972.

7. Williams, G.T., 1994. Programmed cell death - a fundamental protective response to pathogens. Trends Microbiol. 2: 463-464.

8. Roulston, A., R.C. Marcellus, and P.E. Branton, 1999. Viruses and apoptosis. Annu. Rev. Microbiol. 53: 577-628.

9. Simon, H.U., 2003. Targeting apoptosis in the control of inflammation. Eur. Respir. J. 22: 20-21.

10. Saraste, A., and K. Pulkki, 2000. Morphologic and biochemical hallmarks of apoptosis. Cardiovasc. Res. 45: 528-537. 
11. Vaux D.L., and A. Strasser, 1996. The molecular biology of apoptosis. Proc. Natl. Acad. Sci. USA., 93: 2239-2244.

12. Madeo, F., E. Frohlich, M. Ligr, M. Grey, S.J. Sigrist, D.H. Wolf, and K.U. Frohlich, 1999. Oxygen stress: a regulator of apoptosis in yeast. J. Cell Biol. 145: 757-767.

13. Nguewa, P. A., M.A. Fuentes, B. Valladares, C. Alonso, and J.M. Pérez, 2004. Programmed cell death in trypanosomatids: a way to maximize their biological fitness? TRENDS Parasitol. 20: 375380 .

14. Al-Olayan, E. M., G.T. Williams, and H. Hurd, 2002. Apoptosis in the malaria protozoan, Plasmodium berghei: a possible mechanism for limiting intensity of infection in the mosquito. Int. J. Parasitol. 32: 1133-1143.

15. Gareth R. Bicknell, R.T. Snowden, and G.M. Cohen, 1994. Formation of high molecular mass DNA fragments is a marker of apoptosis in the human leukaemic cell line, U937. J. Cell Sci. 107: 2483-2489.

16. Diamond, L. S., D.R. Harlow, and C.C. Cunnick, 1978. A new medium for the axenic cultivation of Entamoeba histolytica and other Entamoebae. Trans. R. Soc. Trop. Med. Hyg. 72: 431-432.

17. Williams, M.S., P.A. Henkart, 1994. Apoptotic cell death induced by intracellular proteolysis. J. Immunol. 153: 4247-4255

18. Huang, T. and A.P. Grollman, 1970. Novel inhibitors of protein synthesis animal cells. Fedn Proc. Fedn. Am. Soc's, Exp. Biol. 29: 609.

19. Goodman, L S. and A. Gilman. 1975. The Pharmacological Basis of Therapeutics, Macmillan Publishins, Co., I NC, New York.
20. Grollman, A.P. 1966. Structural basis for inhibition of protein synthesis by emetine and cycloheximide based on an analogy between ipecac alkaloids and glutarimide antibiotics. Proc Natl Acad Sci U S A. 56: 1867-1874.

21. Chakrabarti, S., D.K. Dube and S.C. Roy, 1972. Effects of Emetine and Cycloheximide on Mitochondrial Protein Synthesis in Different Systems. Biochem. J. 128: 461-462

22. Meijerman, I., W.M. Blom, H.J. de Bont, G.J. Mulder, J.F. Nagelkerke, 1999. Induction of apoptosis and changes in nuclear G-actin are mediated by different pathways: the effect of inhibitors of protein and RNA synthesis in isolated rat hepatocytes. Toxicol. Appl. Pharmacol. 156: 46-55.

23. Cornillon, S., C. Foa, J. Davoust, N. Bounavista and J.D. Gross, 1994. Programmed cell death in Dictyostelium. J. Cell Sci. 107: 2691-2704

24. Nasirudeen, A.M.A., Y.E. Hian, M. Singh and K.S.W. Tan, 2004. Metronidazole induces programmed cell death in the protozoan parasite Blastocystis hominis. Microbiology. 150: 33-43

25. Sen, N., B.B. Das, A. Ganguly, T. Mukherjee, G. Tripathi, S. Bandyopadhyay, S. Rakshit, T. Sen and H.K. Majumder, 2004. Camptothecin induced mitochondrial dysfunction leading to programmed cell death in unicellular hemoflagellate Leishmania donovani. Cell Death Differ. 11: 924-936

26. Halliwel, B. and J.M.C. Gutteridge, 1989. Free radicals in biology and medicine, 2nd. Ed., Clarendon Press, Oxford. 